\title{
ANÁLISIS DE LA PRÁCTICA EDUCATIVA DE LOS MUSEOS PEDAGÓGICOS ESPAÑOLES MEDIANTE UNA EXPERIENCIA DE EVALUACIÓN DE INICIATIVAS, ACTIVIDADES Y RECURSOS
}

\author{
Pablo Álvarez Domínguez \\ Universidad de Sevilla \\ pabloalvarez@us.es \\ María José Rebollo Espinosa \\ Universidad de Sevilla \\ mirebo@us.es
}

\begin{abstract}
RESUMEN
Los museos pedagógicos españoles han venido desarrollando desde su fundación una serie de prácticas educativas que han contribuido de manera determinante (con sus luces y sus sombras) a que hoy sean lo que son. Han emprendido múltiples y diversas iniciativas y actividades para conservar, difundir y ayudar a interpretar el patrimonio educativo. Y de igual forma, cuentan con una gran variedad de recursos que les han permitido llevar a cabo tales funciones. Así, pues, tras una trayectoria educadora más o menos alargada en el tiempo, es el momento de hacer balance. Para responder a este objetivo, hemos utilizado la técnica de evaluación pedagógica Hashtag con un conjunto de miembros de la comunidad de historiadores/as de la educación, interesados especialmente en el estudio del patrimonio educativo, a fin de obtener datos que nos ayuden a esbozar el panorama de las fortalezas, debilidades y anhelos de estos Museos. Los resultados ponen de manifiesto, entre las prácticas consideradas como positivas y transferibles: su uso didáctico, su labor de recuperación y difusión del patrimonio educativo, la colaboración con los centros escolares o el empleo de metodologías alternativas para el aprendizaje de la Historia de la Educación con el alumnado universitario. Las debilidades más repetidas tienen que ver con la falta de personal, espacios y financiación. Y, como propósitos deseables, destacan la necesidad de establecer redes museísticas y planificar proyectos museográficos más interactivos, inclusivos y sociales.
\end{abstract}

Palabras clave: Museos pedagógicos. Práctica educativa. Evaluación.

\section{ANALYSIS OF THE EDUCATIONAL PRACTICE OF SPANISH PEDAGOGICAL MUSEUMS THROUGH AN EXPERIENCE TO EVALUATE INITIATIVES, ACTIVITIES AND RESOURCES}

\begin{abstract}
The pedagogical museums in Spain have developed since their foundation a set of educational practices that have contributed to making them what they are today. These have undertaken multiple and diverse initiatives and activities to conserve, spread and help interpret the educational heritage. And likewise, they have multiple resources that have allowed them to carry out different functions. After a long educational career, it is time to make an assessment. To respond to this objective, we have used the "Hashtag" pedagogical evaluation technique with a group of History
\end{abstract}

Rev. Iberoam. Patrim. Histórico-Educativo, Campinas (SP), v. 6, p. 1-18, e020007, 2020. 
of Education teachers, interested in studying educational heritage. The purpose is to obtain data that helps us point out the strengths, weaknesses and desires of these museums. The results point out as positive and transferable practices: the didactic use of the museum, the work of recovery and dissemination of the educational heritage, collaboration with schools or the use of alternative methodologies for learning the History of Education with university students. The weaknesses are: lack of personnel and space, and little funding. As desirable purposes, they highlight the need to establish museum networks and plan more interactive, inclusive and social museum projects.

Keywords: Pedagogical museums. Educational practice. Evaluation.

\section{ANÁLISE DA PRÁTICA EDUCACIONAL DE MUSEUS PEDAGÓGICOS DA ESPANHA ATRAVÉS DE UMA EXPERIÊNCIA PARA AVALIAR INICIATIVAS, ATIVIDADES E RECURSOS}

\section{RESUMO}

Os museus pedagógicos da Espanha desenvolveram desde a fundação um conjunto de práticas educacionais que contribuíram para torná-los o que são hoje. Estes empreenderam múltiplas e diversas iniciativas e atividades para conservar, disseminar e ajudar a interpretar o patrimônio educacional. E da mesma forma, eles têm vários recursos que lhes permitiram desempenhar funções diferentes. Após uma longa carreira educacional, é hora de fazer uma avaliação. Para responder a esse objetivo, utilizamos a técnica de avaliação pedagógica "Hashtag" com um grupo de professores de História da Educação, interessados no estudo do patrimônio educativo. O objetivo é obter dados que nos ajudem a apontar os pontos fortes, fracos e desejos desses museus. Os resultados apontam práticas positivas e transferíveis: o uso didático do museu, o trabalho de recuperação e disseminação do patrimônio educacional, a colaboração com as escolas ou o uso de metodologias alternativas para o aprendizado da História da Educação com estudantes universitários. Os pontos fracos são: falta de pessoal e espaço, e pouco financiamento. Como finalidades desejáveis, destacam a necessidade de estabelecer redes de museus e planejar projetos de museus mais interativos, inclusivos e sociais.

Palavras-chave: Museus pedagógicos. Prática educacional. Avaliação.

\section{ANALYSE DE LA PRATIQUE ÉDUCATIVE DES MUSÉES PÉDAGOGIQUES ESPAGNOLS À TRAVERS UNE EXPÉRIENCE D'ÉVALUATION DES INITIATIVES, DES ACTIVITÉS ET DES RESSOURCES}

\section{RÉSUMÉ}

Les musées pédagogiques d'Espagne ont développé depuis leur fondation un ensemble de pratiques pédagogiques qui ont contribué à faire d'eux ce qu'ils sont aujourd'hui. Ceux-ci ont entrepris des initiatives et des activités multiples et diverses pour conserver, diffuser et aider à interpréter le patrimoine éducatif. Et de même, ils disposent de multiples ressources qui leur ont permis de remplir différentes fonctions. Après une longue carrière éducative, il est temps de faire un bilan. Pour répondre à cet objectif, nous avons utilisé la technique d'évaluation pédagogique "Hashtag" avec un groupe de professeurs d'histoire de l'éducation, intéressés par l'étude du patrimoine 


\section{$(\mathrm{cc}) \mathbf{E Y}$}

éducatif. Le but est d'obtenir des données qui nous aident à souligner les forces, les faiblesses et les désirs de ces musées. Les résultats indiquent des pratiques positives et transférables: l'utilisation didactique du musée, le travail de récupération et de diffusion du patrimoine éducatif, la collaboration avec les écoles ou l'utilisation de méthodologies alternatives pour l'apprentissage de l'histoire de l'éducation avec des étudiants universitaires. Les faiblesses sont: le manque de personnel et d'espace et peu de financement. À des fins souhaitables, ils soulignent la nécessité d'établir des réseaux de musées et de planifier des projets de musées plus interactifs, inclusifs et sociaux.

Mots clés: Musées pédagogiques, pratique pédagogique, évaluation.

\section{INTRODUCCIÓN / OBJETIVOS}

Haciendo un balance crítico ligado a la historiografía del patrimonio educativo en España en los últimos quince años (LÓPEZ MARTÍN, 2013; MORENO MARTÍNEZ, 2015), podemos reconocer el pronunciado esfuerzo que la comunidad de historiadores/as de la educación está desarrollando en aras de prestar una mayor atención a la memoria social e individual de la educación, derivado de las prácticas, experiencias y vivencias educativas y/o formativas de la ciudadanía. Ciertamente, con ello se justifica -al menos en parte-, la oportunidad de visibilizar en la actualidad nuestro pasado educativo a través de la creación y promoción de museos pedagógicos o centros de memoria educativa (HERNÁNDEZ DÍAZ, 2000; RUIZ BERRIO, 2006).

La reconstrucción del pasado histórico educativo a través de estos espacios pedagógicos ha propiciado una importante labor de estudio, catalogación, organización, exposición y difusión del patrimonio material e inmaterial de la educación. La creación de museos pedagógicos en nuestro país -una trayectoria relativamente consolidada-, ha venido acompañada de notables esfuerzos por hacer de estos espacios atractivos laboratorios de aprendizaje cívico para deleite y disfrute de toda la ciudadanía. No obstante, aunque estemos en disposición de asumir que se ha avanzado mucho en este sentido, también es justo reconocer que aún quedan muchos frentes por explorar y muchas puertas que abrir para hacer de nuestros museos potentes recursos didácticos capaces de desarrollar una función formativa y de utilidad social.

Así, a través de este trabajo, previo análisis teórico somero de lo que son y lo que hacen los museos pedagógicos en España, se pretende dar a conocer sus principales fortalezas, debilidades y retos pendientes en la actualidad, en relación con la gran variedad de iniciativas y actividades que desarrollan y los recursos con los que cuentan. Para ello, utilizaremos la técnica de evaluación 


\section{(cc) $\mathrm{BY}$}

hashtag con la intención de valorar con profesionales de la historia de la educación y el patrimonio educativo, de qué manera la práctica educativa de estos museos pedagógicos se ha convertido en elemento esencial en el desarrollo cultural del conjunto de la sociedad. Desde una perspectiva sociomuseológica (FERNÁNDEZ DOS REIS DIEZ, 2015), analizaremos hasta qué punto estas instituciones contribuyen a compartir con la comunidad su misión conservadora y difusora del patrimonio educativo, ofreciendo actividades y recursos a diferentes grupos y colectivos sociales, que faciliten un diálogo intergeneracional sobre la historia y la memoria de la escuela. Este análisis nos permitirá poner en práctica un sistema de evaluación colaborativa en lo que atañe a problemas y soluciones, mediante el cual -y a través de un estudio cualitativo de carácter interpretativo-, tracemos una aproximación inicial sobre el estado de la cuestión relacionada con el quehacer investigador, docente y de extensión cultural que desarrollan los museos pedagógicos españoles en la actualidad.

\section{LOS MUSEOS PEDAGÓGICOS EN ESPAÑA Y SU PRÁCTICA EDUCATIVA: LO QUE SON Y LO QUE HACEN}

En la obra Los Museos Pedagógicos en España: entre la memoria y la creatividad (ÁLVAREZ, 2016), publicada hace tres años, y en otros trabajos posteriores (ÁLVAREZ 2018; ÁLVAREZ; DÁVILA; NAYA, 2017), se daba cuenta del fuerte impulso que han experimentado estudios, proyectos, iniciativas, etc., sobre el patrimonio educativo en España. Junto a ello, se daba a conocer específicamente un mapeo de los diferentes museos pedagógicos existentes en el país, poniendo de manifiesto su diversidad, estructuras, dependencias, fondos, iniciativas, actividades, recursos, etc. Tales museos, concebidos como instituciones fruto del paradigma de la modernidad ${ }^{1}$, nacen de una conciencia histórica y socioeducadora y de un compromiso institucional y ciudadano con la recuperación del patrimonio y la memoria de la escuela -en comunión con el reciente repensar de una Historia de la Educación más hermenéutica, social y participativa-. Estos museos,

\footnotetext{
${ }^{1}$ La noción de modernidad en este caso, se encuentra ligada a las reflexiones de Clement Greenberg, 2002, y Theodor Adorno, 2009. Clement, que se refiere al concepto de pureza, a una "estética exclusivista", relacionada con la especificidad del medio y de la calidad; y Adorno, que alude al de autonomía (Cfr. GREENBERG, Clement (2002): Arte y cultura: Ensayos críticos. Barcelona: Paidós; ADORNO, Theodor (2009): Crítica de la cultura y sociedad II. Akal: Madrid). Tales conceptos nos ayudan a concebir un museo ubicado entre los años inmediatos a la postguerra y finales de los años sesenta. Ni a uno ni a otro les interesaba la idea de museo contaminado por la propia sociedad industrializada.
}

Rev. Iberoam. Patrim. Histórico-Educativo, Campinas (SP), v. 6, p. 1-18, e020007, 2020. 


\section{(cc) $\overline{E Y}$}

que en España superan los cincuenta (ÁLVAREZ, 2016) -si los consideramos en cuanto a su tipología, por ejemplo-, (ÁLVAREZ; DÁVILA; NAYA, 2017), son concebidos como espacios didácticos vivos en los que una representación de bienes patrimoniales materiales e inmateriales de carácter histórico educativo se dan cita para rendir tributo y consideración a un conjunto de fuentes, vestigios, testigos y testimonios de la Historia de la Educación que contribuyen al desarrollo de nuestra identidad personal y colectiva.

Los museos pedagógicos son espacios que, a caballo entre la memoria del ayer y la invitación a la creatividad que nos envuelve en la actualidad, están respondiendo notablemente al reto de participar activamente en la construcción de una nueva Historia de la Educación Española pública, social, crítica y participativa. Esos museos, más allá de poder ser concebidos como meros espacios expositivos de contenido patrimonial histórico educativo, se presentan como novedosos recursos que nos permiten explorar y documentar visual y textualmente -formal y no formalmente, la historia de diferentes espacios, métodos y recursos de aprendizaje. Un museo pedagógico, tal y como lo entendemos hoy, es un laboratorio socializador de experiencias histórico educativas en las que el patrimonio escolar en diálogo con las personas (estudiantes de Ciencias de la Educación, profesorado, visitantes, etc.), se convierte en elemento dinamizador e inspirador para la interpretación de la historia de la escolarización; es un lugar para la visibilidad de la memoria y de la desmemoria escolar; es un espacio de encuentro con las culturas de la escuela, capaz de inspirar reflexiones y preguntas relacionadas con el modelo educativo al que queremos aspirar; es un territorio mediador (VARINE-BOHAN, 2011) entre la materialidad del pasado educativo y las voces de la inmaterialidad sonora de los testimonios de la escuela; es una estancia expositiva que facilita el aprendizaje, dinamización y proyección cultural de un patrimonio para el que sigue resultando transcendental una sensibilización poblacional inspirada en la necesidad de entenderlo como parte de nuestro legado cultural; es un recinto caleidoscópico de imágenes, formas, textos, palabras, etc., que sin voz nos habla de lugares, espacios y memorias educativas; es un circuito entendido como espacio de representación histórico educativa para ser leído e interpretado culturalmente por una sociedad que no cede suficiente protagonismo a la memoria y que no termina de comprometerse del todo con la visibilidad de la historicidad del hecho educativo; es una respuesta en sí misma, a lo que los estudiosos del patrimonio escolar han ideado como galerías abiertas para aglutinar dignamente la temporalidad de la Historia de la Educación, a través de escenas características y piezas que ilustran sus cambios y sus rupturas. 


\section{(cc) EY}

Y además de lo que estos museos son, no cabe duda de que su protagonismo les viene dado por lo que hacen y por lo que serán capaces de hacer a corto, medio y largo plazo. En este sentido, son destacables las iniciativas y actividades que desarrollan en relación con el patrimonio educativo, y que tienen que ver - principalmente-, con las siguientes cuestiones (ÁLVAREZ, 2016): localización, inventariado y conservación; exposición, exhibición, interpretación y extensión cultural; difusión, puesta en valor y divulgación; etc. En definitiva, en la medida en que desde los museos pedagógicos se mezclan memorias del pasado con experiencias presentes y anhelos para la educación del futuro, los estamos convirtiendo, como señala Grosvenor (2019), en lugares de aprendizaje pensados como artificios para ser observados y para que las personas observadoras aprendan que las historias del aprendizaje pasado son las que nos llevarán a comprender la realidad educativa de los tiempos que corren.

De esta forma, conocer las prácticas educativas en el pasado, implementadas tanto dentro de la institución escolar como fuera de ella, requiere de la consideración y uso de determinadas fuentes que nos permitan interpretar los registros tangibles de esta práctica, tales como mobiliario, objetos, espacios, materiales e instrumentos didácticos, manuales escolares, etc., y que nos informen a la vez sobre tales prácticas, a través de memorias docentes y de actividades, memorias del alumnado, memorias de oposición, informes, apuntes de clase, cuadernos escolares, testimonios personales, fotografías, álbumes, revistas escolares, anuarios, trabajos de estudiantes, etc. Ante esta tarea, el trabajo que se viene desarrollando desde los museos pedagógicos españoles -con sus luces y sus sombras-, resulta fundamental para que el estudio y la conservación de la memoria y la práctica educativa -en estrecha interrelación-, nos ayude a visibilizar indicios que interpretados nos permitan reconstruir las realidades vividas a lo largo del tiempo educativo.

\section{MÉTODO: LA TÉCNICA HASHTAG PARA EVALUAR LA PRÁCTICA EDUCATIVA DE LOS MUSEOS PEDAGÓGICOS ESPAÑOLES}

Tras una trayectoria investigadora y educadora más o menos alargada en el tiempo, los museos pedagógicos españoles tienen en la actualidad la obligación de hacer un balance evaluativo que contribuya a poner de manifiesto cuánto han hecho, están haciendo y tienen pendiente hacer en relación con su práctica educativa. En este sentido, se entendió que las VIII Jornadas Científicas de la Sociedad Española para el Estudio del Patrimonio Histórico Educativo (SEPHE): "La Práctica 


\section{$(\mathrm{cc})$ EY}

Educativa: Historia, Memoria y Patrimonio", celebradas en Palma de Mallorca (España) en noviembre de $2018^{2}$, se presentaba como un escenario adecuado para evaluar cuantas iniciativas y actividades han emprendido y están emprendiendo estos museos, así como los recursos con los que han contado y cuentan para el ejercicio de su actividad cotidiana. Y esto, principalmente, porque en un mismo lugar se congregarían un amplio número de profesionales vinculados al quehacer educativo de esta tipología de museos. Así, en el marco de estas jornadas, se planteó el taller titulado: "Museos pedagógicos y práctica educativa: la reconstrucción de la práctica educativa en España a través de sus museos pedagógicos: recursos, posibilidades e iniciativas", bajo la coordinación de los profesores Pablo Álvarez Domínguez y Teresa Rabazas Romero, en el cual representantes de seis Museos españoles ${ }^{3}$ intercambiaron en formato de mesa redonda, sus experiencias concretas en torno a la temática que nos había reunido. La pretensión final de este taller se centraba en la obtención de una serie de datos que nos ayudaran a esbozar el panorama de las principales fortalezas, debilidades y anhelos educativos a los que aspiran los museos pedagógicos españoles. Como colofón del mismo y para responder a este objetivo, utilizamos la técnica de evaluación pedagógica Hashtag con un grupo de 34 personas, siendo conscientes de sus limitaciones, sin que se complemente con el uso de otras técnicas evaluativas. Entre los participantes, se encontraban gestoras o personal vinculado a museos pedagógicos, así como otras personas interesadas especialmente en el estudio del patrimonio educativo.

2 Cfr. http://www.sephe2018.com/15493/detail/viii-jornades-cientifiques-de-la-sephe-i-congresso-nazionale-dellasipse.html Estas jornadas coincidieron con la celebración del I Congresso Nazionale della SIPSE.

${ }^{3}$ En el taller intervinieron con ponencias representantes de los siguientes museos pedagógicos: Universidad de Sevilla, Universidad de Huelva, Universidad del País Vasco, Universidad Complutense de Madrid, Universidad de La Laguna y AMEIB. 


\section{$(\mathrm{cc})$ EY}

FOTOGRAFÍA 1 - (De izquierda a derecha). Los profesores Alejandro Mayordomo Pérez,

Pablo Álvarez Domínguez y Teresa Rabazas Romero durante el desarrollo del taller "Museos pedagógicos españoles y práctica educativa" en las VIII Jornadas Científicas de la SEPHE. Palma de Mallorca, noviembre de 2018.

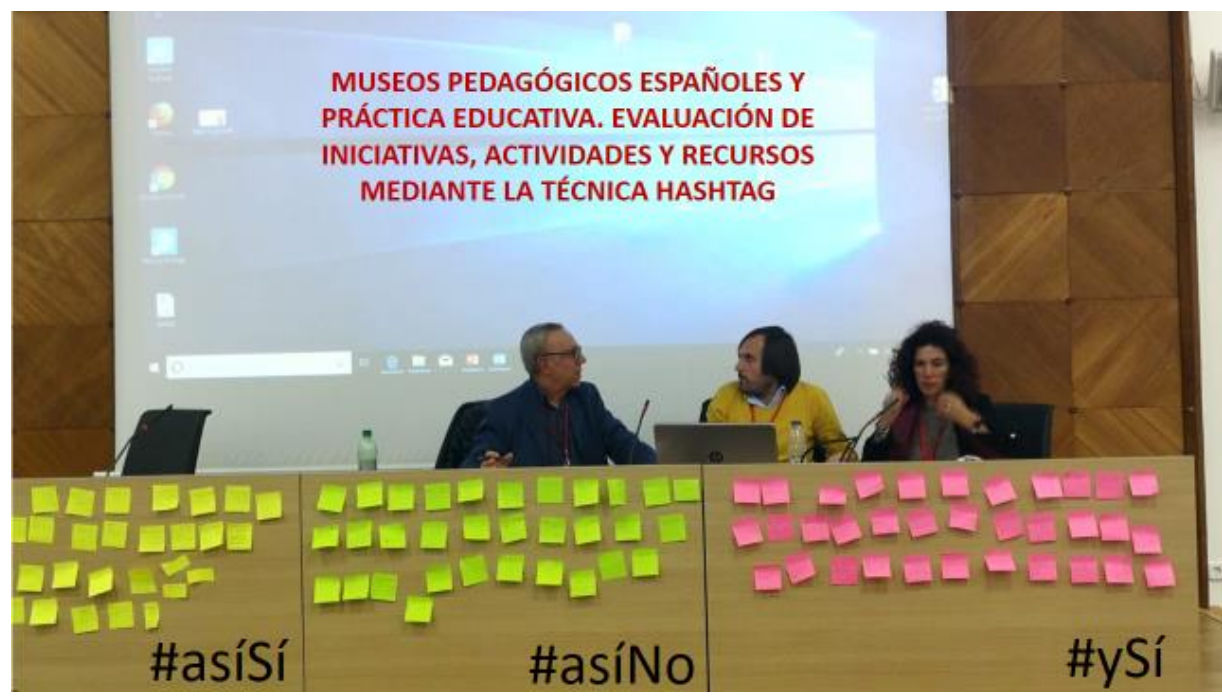

Fuente: Archivo del autor.

La técnica de evaluación hashtag es una nueva técnica evaluativa, participativa y creativa que, basada en el pensamiento paralelo de Edward de Bono (2007), han ideado los profesores Herrero-Vázquez y Torres-Gordillo (en prensa). Está inspirada en la red social Twitter y el uso del emblemático hash (almohadilla), aprovechando el tirón de esta red social para desdramatizar y socializar la evaluación de una manera más colaborativa. Así, los factores evaluables están relacionados con 3 aspectos fundamentales: a) con lo que ha parecido idóneo; b) con lo que no se está de acuerdo; y c) con propuestas creativas de cara al futuro. El apelativo hashtag de esta técnica evaluativa responde a la incorporación de la almohadilla (\#) a las etiquetas (tag): asíSí, asíNo y ySi. En nuestro caso, para mejorar la eficiencia de un taller de apenas 2 horas de duración, en lugar de utilizar la red social, hicimos uso de pósits de 3 colores diferentes, y a cada uno de los participantes se le dio uno de cada color para que, en cada uno de ellos, respondieran anónimamente a las siguientes preguntas: \#asiSI: ¿qué están haciendo bien los museos pedagógicos?; \#asiNo: ¿qué no se están haciendo del todo bien?; \#ySi: ¿qué cosas nuevas se podrían hacer a modo de sugerencias de mejora?

Las diferentes respuestas recogidas fueron dadas a conocer públicamente en el taller, y sobre ellas, se plantearon diferentes comentarios, reflexiones e interrogantes. Sin embargo, al no 


\section{$(c)$ EY}

ser posible en el momento un análisis más exhaustivo de las respuestas vertidas, y una vez finalizado el taller, optamos tiempo después por realizar un análisis de datos de corte cualitativo. Dado que no se trata de un cuestionario cerrado, sino todo lo contrario, con total libertad creativa, es muy raro que se repitan respuestas, hecho que dificulta muchísimo la cuantificación o la mera contabilización de ideas coincidentes. De ahí que la tarea de organización e interpretación de los dispersos datos obtenidos resulte compleja y se preste a niveles de subjetividad relativos Semejante problema queda compensado con las dosis de espontaneidad e intuición, exigidas por la técnica en sí, que valora la rapidez de las contestaciones, puesto que esta garantiza que salgan a la luz cuestiones importantes, sin pasar casi por el filtro de lo racional, convencionalmente establecido o políticamente correcto ${ }^{4}$. Nuestra labor de análisis consistirá pues, fundamentalmente, en ordenar la información recibida a modo de brainstorming, para interpretar y deducir lo que el colectivo evaluado opina y propone.

Para ello, hemos sometido cada una de las aportaciones a reflexión, agrupando las que estaban en una línea similar y colocándolas según orden de preferencia, en función del número de veces que aparecían reflejadas en las respuestas. Se han mantenido como categorías de análisis, los tres hastags iniciales:

TABLA 1- Categorías de análisis. Descripción de categorías.

\begin{tabular}{|c|l|}
\hline CATEGORÍAS DE ANÁLISIS & \multicolumn{1}{|c|}{ DESCRIPCIÓN } \\
\hline \#asíSí (Fortalezas) & $\begin{array}{l}\text { Prácticas que los museos reconocen como positivas, } \\
\text { cosas que les funcionan, elementos que se entienden } \\
\text { como fortalezas. }\end{array}$ \\
\hline \#asíNo (Debilidades) & $\begin{array}{l}\text { Prácticas que han fallado, que se han mostrado poco } \\
\text { acertadas o cuestiones que se echan en falta, carencias } \\
\text { detectadas como debilidades, errores u obstáculos que } \\
\text { convendría subsanar. }\end{array}$ \\
\hline \#ySi (Oportunidades) & $\begin{array}{l}\text { Prácticas deseadas, cosas que se querrían conseguir } \\
\text { pero quedan por hacer, factores que se entiende que }\end{array}$ \\
\hline
\end{tabular}

\footnotetext{
${ }^{4}$ Solo se les limitó el tiempo para responder, puesto que así lo determina la técnica utilizada, dado que es justamente la obligación de ofrecer las respuestas que más rápidamente se nos ocurren, lo que hace que afloren los temas de forma más intuitiva, sincera y franca, punto de partida o materia prima para trabajarlas con posterioridad a un nivel más teórico y racionalizado.
}

Rev. Iberoam. Patrim. Histórico-Educativo, Campinas (SP), v. 6, p. 1-18, e020007, 2020. 


\begin{tabular}{|l|l|}
\hline & $\begin{array}{l}\text { optimizarían las potencialidades del museo, } \\
\text { propuestas de mejora. }\end{array}$ \\
\hline
\end{tabular}

Fuente: Elaboración propia.

\section{ANÁLISIS DE RESULTADOS}

A continuación, ordenamos los datos, considerando las respuestas obtenidas, con el fin de extraer y visibilizar iniciativas, actividades y recursos como patrones de referencia, consecuencias útiles para marcar estrategias futuras, a partir de experiencias museístico-pedagógicas y aprendizajes compartidos.

En relación con la primera categoría de análisis, FORTALEZAS (\#asíSî), las respuestas más repetidas tienen que ver con los usos didácticos de los museos pedagógicos. Parece ser que la mayoría de nuestros museos prioriza claramente sus labores educativas, como no podía ser menos al tratarse de este tipo de instituciones museísticas. También han entendido y aprovechado las potencialidades que tienen como espacios de educación informal, siendo capaces de despertar aprendizajes significativos en audiencias de todo tipo. Así, varios de los informantes subrayan su operatividad a la hora de ofrecer una enseñanza complementaria de calidad especialmente de cara al alumnado universitario, sobre todo al que cursa itinerarios de magisterio o grado en pedagogía. Pero además están desarrollando buenas prácticas destinadas a los grupos de escolares: las visitas de estudiantes de infantil, primaria y secundaria al museo tienen muy buena acogida, más aún si se adaptan a la edad del público a base de talleres y actividades específicas y si se planifican preparando una introducción previa en el aula y un trabajo posterior post-visita de vuelta al aula, para afianzar aprendizajes y extenderlos a otros ámbitos (como la familia, por ejemplo). Igualmente, no podemos olvidar el éxito que suelen tener las visitas de mayores (aulas de la experiencia, residencias, centros de día, asociaciones municipales, etc.), una audiencia cuyo protagonismo es más patente en las aulas del pasado recreadas, puesto que son las suyas de la infancia; un público del que se aprende muchísimo, porque les ponen sus voces, experiencias y sentimientos a los objetos expuestos; un público, además, que puede fomentar las relaciones intergeneracionales si las visitas se programan uniendo mayores y jóvenes en los grupos. No obstante, debido a que los mayores no forman parte del sistema educativo en sí mismo, no siempre es fácil llegar a los mismos a través de los recursos comunicativos (CALAF; FONTAL, 2004) con 


\section{$(c)$ EY}

los que cuentan los museos pedagógicos. Sin embargo, tenemos que ser capaces de aprovechar el potencial de las nuevas tecnologías de la información y la comunicación (SANTACANA; LÓPEZ, 2015) (experiencias de realidad virtual, actividades interactivas, etc.), para acercarnos a los mencionados.

Asimismo, nuestros museos se muestran satisfechos por el adecuado cumplimiento de las otras funciones clásicas, siempre presentes en los reglamentos de estos organismos: la recuperación, difusión e investigación del patrimonio histórico-educativo. Reconocen, pues, llevar a cabo, en la medida de sus posibilidades, estas tareas imprescindibles: conservan y protegen los bienes relacionados con el ajuar escolar o los procesos educativos en general, los exponen y les dan visibilidad, para sensibilizar al público y ponerlos en valor, y promueven que se continúe investigando a través de ellos para reconstruir la Historia de la Educación, como recomiendan las modernas tendencias historiográficas, en el sentido planteado por Moreno Martínez (2015).

Por último, aún entre las fortalezas, señalar que nuestra comunidad museística defiende la idea de que no hay que quedarse anclados en el pasado. Partidarios del nuevo paradigma conceptual ligado al museísmo pedagógico (ÁLVAREZ DOMÍNGUEZ, 2010), hay quienes los conciben como espacios lúdicos, vivos, cuidando la estética museográfica. De ahí que la mayoría se decante por el uso de recreaciones de aulas pasadas, que incentivan la interpretación y la memoria. Y de ahí también que intenten ser interactivos, mediante el diseño de actividades que impliquen la participación o, al menos, permitiendo la manipulación de los objetos, algo que en los museos tradicionales era inconcebible. Además, apuestan por unir pasado y presente gracias a la aplicación de las nuevas tecnologías de la información y comunicación (BELLIDO GANT, 2001), que amplían el horizonte de discurso y difusión de los museos. Y, al hacer todo esto, se están sentando las bases para la práctica de una necesaria educación patrimonial: este tipo de museos, mucho más abiertos a la sociedad, fomentan y consolidan valores que contribuyen a formar una ciudadanía más democrática.

En lo que respecta a la segunda de las categorías de análisis, a las DEBILIDADES (\#asíNo), se describen, de un lado, los problemas confesados y, de otro, las carencias manifestadas. El problema que aparece mencionado en más ocasiones tiene que ver con el espacio. Un par de museos se quejan de las limitaciones de sus espacios físicos, es decir de la exigüidad de sus lugares de exposición y trabajo. Pero, con mucho, es el espacio simbólico el que preocupa: vemos planteado de varias maneras el hecho de que los museos pedagógicos son una isla dentro de la propia 


\section{(cc) $\mathbf{E Y}$}

universidad, facultad, o lugar en el que se emplazen. Dos participantes utilizan el término "encerrados", para destacar el hermetismo que presentan. Éste puede tener que ver con los problemas de horario de apertura que muchos sufren (franjas muy cortas de visita o solo visitas solicitadas previa cita y programadas con mucha antelación, por falta de personal que las atienda) y los mantiene literalmente cerrados al público. Pero eso, a su vez, hace que aumente su invisibilidad ante la comunidad educativa que debería ser su audiencia potencial: en los universitarios, por ejemplo, con frecuencia, el propio alumnado de la facultad (y todavía con más razón el público externo) desconoce su existencia, pasan desapercibidos, no se hacen ver. Entonces estaríamos hablando de un fallo en la difusión, en la comunicación eficaz entre instancias, en la diversidad de propuestas que se diseñan para los diferentes públicos o incluso de un problema de falta de confianza en lo que respecta a tomar conciencia de las posibilidades que tienen los museos pedagógicos para la transferencia de conocimientos.

Otro problema, en parte conectado con el anterior, hace referencia a la poca conexión con la juventud (BECERRA; DOMÍNGUEZ, 2014) para la que, al menos en teoría, los museos pedagógicos fueron concebidos en gran medida. Demasiadas veces, cuando se reciben visitas de jóvenes, se trata del llamado "público cautivo", o sea, de personas que acuden obligadas al museo, como un elemento más en la programación de sus actividades curriculares o una propuesta incluida en una agenda de excursiones prefijada por otros, pero nunca como una iniciativa voluntaria, resultado de un interés personal hacia lo que en él se pueda ver o aprender. Habría que plantearse, en consecuencia, llevar a cabo pertinentes estudios de público (URGELL PLAZA, 2014), a fin de buscar nuevas maneras de captarlo, quizá hablando en un lenguaje más cercano al suyo u ofreciéndole propuestas más en su onda generacional y motivacional.

También se apuntan como problema las dificultades que lleva aparejada la adaptación de los museos a toda clase de públicos, incluidas las personas con alguna discapacidad (motora, sensorial, mental) o necesidad educativa especial, es decir, el reto de la inclusión y la accesibilidad (ESPINOSA RUÍZ; BONMATÍ LLEDÓ, 2014). Seguramente porque esta tarea, ineludible por equidad y justicia, se contempla como un trabajo extra, añadido a la "cotidianeidad" del museo o, quizá también, porque se reconoce una preparación escasa en este terreno tan delicado y complejo, para el que se requiere, además de sensibilidad y empatía, una formación específica.

Y otros declaran, como problema, las dificultades a la hora de dejar atrás el cómodo concepto de museo tradicional, que gira en torno a la exposición de los fondos en vitrinas cerradas, 


\section{(cc) $\mathrm{EY}^{\mathrm{B}}$}

manteniendo los materiales fuera del alcance del público porque se prima ante todo la conservación. Se comenta, asimismo, en esta línea, la indefinición teórica en la que a menudo nos movemos en nuestra área, y la falta de referentes metodológicos que ayuden a pasar de un museo de "cosas muertas" a un museo nuevo, como mandan los cánones de la museología actual. Esta inseguridad provoca a veces la sensación, o la constatación, de que los que se consiguen en los museos pedagógicos no son aprendizajes de calidad, sino superficiales, llegándose incluso a repetir en ellos estereotipos históricos y educativos que deberían ser obviamente descartados.

Entre las carencias resaltan claramente dos: de personal y de financiación. La falta de recursos humanos es un tópico de lo más preocupante, porque puede impedir con facilidad la marcha del museo. Se echa en falta un personal fijo, no transitorio (como sería el caso del alumnado en prácticas o estudiantes colaboradores/internos, que pasan por el museo tan solo un par de meses) que complemente el voluntarismo al que se ven abocadas las personas responsables de los museos, que asumen las obligaciones generadas por éstos aparte de las docentes e investigadoras y sin ningún tipo de incentivo académico o crematístico. Se piden como mínimo becarios/as que permitan mantenerlo abierto y productivo en horario fijo, a fin de evitar la invisibilidad de la que hablábamos antes. Pero se solicita, con mayor insistencia aún, la colaboración de personal técnico preparado, para tener al día labores básicas como la catalogación y digitalización de los fondos, que a menudo se desatiende por este motivo.

Y, en cuanto a la falta de financiación, solo decir que es otra de las denuncias más unánimes. Es un común denominador que afecta prácticamente a todos los museos pedagógicos, a pesar de que la situación económica o las soluciones arbitradas a este respecto sean bastante dispares entre ellos. Los medios se suelen calificar de precarios y se denota la ausencia de apoyo institucional o de implicación por parte de la Administración competente. Paradójicamente, alguno de los museos que sí cuenta con fondos públicos ve condicionadas sus actividades por esta dependencia. Pero, en general, la captación de recursos económicos sigue siendo una asignatura pendiente.

Y en cuanto a la categoría de OPORTUNIDADES (\#ySI), en el plano de los desafíos por conseguir o propuestas de mejora, encontramos uno que podría abarcar sintéticamente a todos los demás: el deseo y empeño por modernizar, por cambiar de verdad el tipo de museo, logrando, entre otras cosas, que los museos pedagógicos sean más:

- Conectados. La palabra "redes" ha sido la más elegida en este apartado. Se demanda la creación de redes museísticas a todos los niveles (de centros históricos, de museos pedagógicos 


\section{$(c c)$ EY}

nacionales, internacionales...), que faciliten el intercambio de experiencias y hagan posible el trabajo en colaboración de una comunidad científica que comparte, como estamos viendo, multitud de circunstancias y puede apoyarse mutuamente para aprender de forma cooperativa. Se concretan incluso algunas tareas encaminadas a la confección y funcionamiento de dichas redes, por ejemplo, la digitalización de los fondos, la construcción de una base de datos unificada o el impulso a las estancias de investigación y docencia en museos pedagógicos de otras ciudades o países.

- Conocidos. Con el objetivo de cubrir una de las deficiencias actuales señalada más arriba, la del desconocimiento que suele tener la ciudadanía acerca de los museos pedagógicos, se propone profundizar en las labores de difusión y divulgación, ya sea a través de los medios de comunicación de masas y las redes sociales, o bien orquestando estrategias de marketing (MARTÍNEZVILANOVA, 2017), campañas informativas en los centros educativos, o editando publicaciones monográficas sobre patrimonio histórico-educativo.

- Abiertos, accesibles, inclusivos, sociales. Un alto número de participantes entiende que los museos tienen que comprometerse a abrir sus puertas, a solventar ese hermetismo que decíamos parece haberlos caracterizado hasta ahora en determinados casos. Quieren aprovechar las oportunidades que les brinda el recibir visitas escolares, por ejemplo, pensando en realizar un trabajo mucho más intenso y continuado con las escuelas para desarrollar en la práctica programas de educación patrimonial más efectivos. Apuestan por la interdisciplinariedad, por el trabajo en equipos de especialistas de diferentes campos complementarios. Y manifiestan también su deseo de colaborar con otras instituciones sociales y culturales, en lugar de levantar muros que desubiquen y entierren a los museos en su propia mismidad.

- Atractivos. Como una vía más para ganarse a su público, los museos son conscientes y están dispuestos a mejorar su imagen y a responder a las demandas e intereses de sus usuarios/as potenciales. Para ello, varias personas coinciden en sugerir que se preste más atención a fondos museísticos distintos de los más habituales (objetos materiales, documentos, manuales escolares), por ejemplo, los iconográficos y, sobre todo, se reivindica la elaboración de bancos de historia oral. Repiten igualmente que un museo debe ser mucho más que una mera sala de exposiciones: tiene que convertirse en un espacio para potenciar ilusiones, revivir emociones, jugar con todos los sentidos e incentivar la experimentación interactiva y el disfrute.

- Organizados. Se reconoce que las tareas desempeñadas por estos museos tendrían que estar mejor planificadas. Trabajando en equipos interdisciplinares, como se aconsejaba con 


\section{(cc) $\mathbf{E Y}$}

anterioridad, los museos no dejarían tanto al azar, a la improvisación o a la repetición mecánica de sus acciones, sino que, a corto, medio y largo plazo diseñarían proyectos museológicos y museográficos por los que guiarse. Y un importante elemento, muy olvidado, completaría dichos proyectos, la evaluación, un factor de retroalimentación y reajuste imprescindible.

- Científicos. Bajo este adjetivo queremos recoger la idea de que se espera activar los museos como centros de investigación de la cultura material e inmaterial de la escuela, lo cual pasa necesariamente por aumentar la participación de estudiantes universitarios en esta área disciplinar de la Historia de la Educación.

- Amplios. Por último, seguramente los representantes de los museos más pequeños, como es lógico, anhelan espacios físicos más grandes, más metros cuadrados para poder, por ejemplo, recrear aulas de distintas épocas que permitan comprobar la evolución de la escenografía escolar, los materiales didácticos, las metodologías, los contenidos, las doctrinas pedagógicas o los discursos ideológicos sostenidos por las políticas educativas cambiantes. O para disponer también de zonas suficientemente preparadas para el trabajo interno que exige este tipo de instituciones (catalogación, investigación, diseño de actividades, etc.). En ocasiones, las limitaciones físicas han hecho derivar a los museos hacia soluciones complementarias o suplementarias, como los espacios virtuales interactivos, que, además de superar ese obstáculo, quizá logren captar a otro tipo de público, el nativo de la era digital.

\section{A MODO DE CONCLUSIÓN: CONSIDERACIONES FINALES}

Atendiendo a lo expuesto por quienes han participado en esta evaluación mediante hastag, retomemos nuestras preguntas iniciales: ¿cuáles son, pues, las buenas prácticas museísticas que deberíamos seguir ensayando?, ¿qué errores convendría evitar o qué problemas tendríamos que empeñarnos en intentar solucionar?, ¿y hacia dónde habría que dirigir las futuras estrategias para mejorar la calidad educativa y las funciones sociales aparejadas a los museos pedagógicos?

Los resultados -limitados eso sí-, a las circunstancias particulares de los participantes en el proceso de evaluación, ponen de manifiesto entre las prácticas consideradas como positivas y transferibles: su uso didáctico, en los terrenos de la educación formal, no formal e informal, dentro y fuera de la universidad, empleando metodologías alternativas para el aprendizaje de la Historia de la Educación, con la finalidad de fomentar una educación patrimonial que construya ciudadanía 


\section{$(c)$ EY}

democrática; y sus labores de recuperación, difusión e investigación sobre el patrimonio educativo, potenciadas en gran medida gracias a que son concebidos cada vez más como centros de interpretación participativos.

Las debilidades más repetidas tienen que ver con la falta de personal, espacios y financiación. Se denuncia especialmente la escasez de personal fijo dedicado en exclusiva a los museos y bien formado en las técnicas museísticas y museográficas. Existe una queja bastante compartida acerca de la precariedad de los espacios y la poca visibilidad, presencia y apertura de los museos pedagógicos de cara a la comunidad. Y los presupuestos resultan casi siempre cortos, cuando no inexistentes. Además, se reconocen las dificultades que entraña la conversión en museos modernos, interactivos, inclusivos y accesibles, capaces de atraer a un público joven de forma voluntaria.

Y, como propósitos deseables, destacan la necesidad de establecer redes museísticas y planificar proyectos museográficos que trabajen precisamente en la línea de modernización de los museos. Divulgación eficaz, acercamiento a potenciales visitantes, uso de lenguajes actuales (TIC), servicio a la sociedad, respeto a la diversidad, interdisciplinariedad, innovación, apuesta por lo lúdico, atención a las emociones y la experimentación con los sentidos, evaluación a medida, reconocimiento por parte de otras instancias educativas... son algunos de los ingredientes que se apuntan como necesarios para que funcione la fórmula de un museo pedagógico acorde con los tiempos y capaz de sacar el máximo partido posible al patrimonio histórico-educativo, como fuente para reconstruir y dar a conocer una Historia de la Educación crítica, reflexiva y conectada con la realidad presente.

En definitiva, los museos pedagógicos deberían modificar sus modelos y procesos, modernizar sus métodos de trabajo, diseñar y utilizar mecanismos de evaluación para mejorar y extender las buenas prácticas, y buscar el reconocimiento social a través de la participación de todas las personas interesadas de forma compartida. Porque no podemos permitir que nuestros museos de Educación se anquilosen y se conviertan en refugios cerrados exclusivamente para la comunidad de historiadores de la educación y sus estudiantes. Y tampoco podemos quedarnos parados en un tiempo que ya pasó y seguir sobreviviendo entre el polvo de las vitrinas limitándonos a conservar, exponer y enseñar nuestras raíces histórico-educativas, al margen de la complejidad y progreso que la misma sociedad nos impone. Si pretendemos contribuir a la difusión de esta faceta tan fundamental de la cultura que es la Historia de la Educación, habrá que plantearse con ahínco 


\section{(cc) $\mathbf{E Y}$}

y grandes dosis de creatividad e imaginación maneras de mantenerla viva dentro de las permeables y acogedoras paredes de los museos pedagógicos, donde interactuar con las evidencias y señales de los objetos y con las voces de los y las protagonistas de los procesos educacionales será la clave.

Finalmente, conscientes de que estas conclusiones se circunscriben solamente a una experiencia de evaluación puntual, habría que señalar que podemos entenderla como un punto de partida para realizar estudios en profundidad en esta línea con otras técnicas complementarias que ayuden a responder con mayor fiabilidad a las cuestiones que en este trabajo nos hemos planteado.

\section{BIBLIOGRAFÍA}

ADORNO, Theodor. Crítica de la cultura y sociedad II. Madrid: Akal, 2009.

ÁLVAREZ DOMÍNGUEZ, Pablo. Nuevos concepto de los museos de educación. En: RUIZ BERRIO, Julio (ed.). El patrimonio histórico-educativo. Su conservación y estudio. Madrid: Biblioteca Nueva, 2010. p. 139-168.

ÁLVAREZ DOMÍNGUEZ, Pablo (Coord.). Los Museos Pedagógicos en España: entre la memoria y la creatividad. Gijón: TREA y EUS, 2016.

ÁLVAREZ DOMÍNGUEZ, Pablo. Mapping the Museology of Education in Spain: an examination of where the issue currently sltands. Historia da Educaçao, v. 22, n. 55, p. 293-313, 2018.

ÁLVAREZ DOMÍNGUEZ, Pablo; DÁVILA BALSERA, Paulí; NAYA GARMENDIA, Luís María. Education museums: historical educational discourse typology and characteristics. The case of Spain. Paedagogica Historica, v. 53, n. 6, p. 827-845, 2017.

BECERRA, Elena; DOMÍNGUEZ, Beatriz. Museos, comunicación y jóvenes: la comunicación y sus efectos en la población de referencia del museo. Historia y comunicación social, v. 19, n. Esp., p. 603-611, Enero 2014.

BELLIDO GANT, Mª Luisa. Arte, museos y nuevas tecnologías. Gijón: TREA, 2001.

CALAF, Roser; FONTAL, Olaia (Coords.). Comunicación educativa del patrimonio: referentes, modelos y ejemplos. Gijón: TREA, 2004.

DE BONO, Edward. El pensamiento lateral: manual de creatividad. Barcelona: Paidós, 2007. 


\section{$(\mathrm{cc}) \mathbf{E Y}$}

ESPINOSA RUÍZ, Antonio; BONMATÍ LLEDÓ, Carmina. Manual de accesibilidad e inclusión en museos y lugares del patrimonio cultural y natural. Gijón: TREA, 2014.

FERNÁNDEZ DOS REIS DIEZ, Ana Mercedes. La creación de valor en el museo y la sociomuseología. Complutum, v. 26, n. 2, p. 199-206, 2015.

GREENBERG, Clement. Arte y cultura: Ensayos críticos. Barcelona: Paidós, 2002.

GROSVENOR, Ian. Las ciudades del aprendizaje en tiempos modernos. En: Madrid, ciudad educadora (1898-1938). Memoria de la Escuela Pública. Ensayos en torno a una exposición. Madrid: Ayuntamiento de Madrid. Oficina de Derechos Humanos y Memoria, 2019.

HERNÁNDEZ DÍAZ, José María. Los museos de educación en España. En: FERNÁNDES, Rogério e LOURO FELGUEIRAS, Margarida (orgs.): A Escola Primária: entre a imagem e a memoria. Porto: Projecto Museu Vivo da Escola Primária, 2000. p. 94-104.

HERRERO-VÁZQUEZ, Eduardo A.; TORRES-GORDILLO, Juan Jesús. 69 técnicas creativas para la docencia. Sevilla: EUS, (en prensa).

LÓPEZ MARTÍN, Ramón. Historia de la escuela y cultura escolar: dos décadas de fructíferas relaciones. La emergente importancia del estudio sobre el patrimonio escolar. Cuestiones Pedagógicas, 22, p. 17-42, 2013.

MARTÍNEZ-VILANOVA, Rafael. Realidad y posibilidades del marketing en los museos de España. Gijón: TREA, 2017.

MORENO MARTÍNEZ, Pedro Luís. La historiografía del patrimonio educativo en España: un balance crítico. Educar em Revista, 58, p. 87-102, 2015.

RUIZ BERRIO, Julio. Historia y Museología de la Educación. Despegue y reconversión de los Museos Pedagógicos. Historia de la Educación, Revista Interuniversitaria, 25, p. 271-290, 2006.

RUIZ BERRIO, Julio (Ed.). El patrimonio histórico-educativo. Su conservación y estudio. Madrid: Biblioteca Nueva, 2010

SANTACANA, Joan y LÓPEZ, Victoria (Coords.). Educación, tecnología digital y patrimonio cultural. Para una educación inclusiva. Gijón: TREA, 2015.

URGELL PLAZA, Ferrán. Manual de estudios de público de museos. Gijón: TREA, 2014.

VARINE BOHAN, Hugues. El museo mediador. Revista de museología, n. 49, p. 16-23, 2011.

Recebido em: 05 de maio de 2020 Aceito em: 09 de junho de 2020 